\title{
Nonstationary Fronts in the Singularly Perturbed Power-Society Model
}

\author{
M. G. Dmitriev, ${ }^{1}$ A. A. Pavlov, ${ }^{2}$ and A. P. Petrov ${ }^{3}$ \\ ${ }^{1}$ Institute of System Analysis of RAS, Higher School of Economics, Moscow 101000, Russia \\ ${ }^{2}$ Russian State Social University, Moscow 129226, Russia \\ ${ }^{3}$ Keldysh Institute of Applied Mathematics RAS, Moscow 125047, Russia
}

Correspondence should be addressed to M. G. Dmitriev; mdmitriev@mail.ru

Received 23 July 2013; Accepted 15 September 2013

Academic Editor: Allaberen Ashyralyev

Copyright (c) 2013 M. G. Dmitriev et al. This is an open access article distributed under the Creative Commons Attribution License, which permits unrestricted use, distribution, and reproduction in any medium, provided the original work is properly cited.

The theory of contrasting structures in singularly perturbed boundary problems for nonlinear parabolic partial differential equations is applied to the research of formation of steady state distributions of power within the nonlinear "power-society" model. The interpretations of the solutions to the equation are presented in terms of applied model. The possibility theorem for the problem of getting the solution having some preassigned properties by means of parametric control is proved.

\section{Introduction}

Since the work [1], the theory of contrasting structures has become one of the most booming areas of research of the singularly perturbed differential equations [2-4].

The contrasting structures having the form of nonstationary fronts for parabolic partial differential equations were studied in [5]. The theory was applied to propagation of magnetic fronts in spiral galaxies [6-8]. Here we consider the nonstationary fronts in the Mikhailov "power-society" model [9-12] and the possibility to control them.

In the most general case the "power-society" model has the form of a Neumann boundary value problem for nonlinear parabolic integrodifferential equation. In the absence of some political mechanisms the model is reduced to singularly perturbed parabolic Neumann boundary value problem:

$$
\begin{gathered}
\frac{\partial p}{\partial t}=\varepsilon^{2} \frac{\partial^{2} p}{\partial x^{2}}+F(p, x), \\
\left.\frac{\partial p}{\partial x}\right|_{x=0}=\left.\frac{\partial p}{\partial x}\right|_{x=1}=0, \\
p(x, 0)=p^{0}(x) .
\end{gathered}
$$

Here $\varepsilon \ll 1$ is a small positive parameter. Within the "powersociety" model this parameter is small if the hierarchy is long or if society is strong.

The steady-state problem has the form

$$
\varepsilon^{2} \frac{\partial^{2} p}{\partial x^{2}}+F(p, x)=0,\left.\quad \frac{\partial p}{\partial x}\right|_{x=0}=\left.\frac{\partial p}{\partial x}\right|_{x=1}=0 .
$$

Let the following conditions hold [2-4].

(1) The function $F(p, x)$ has continuous partial derivatives for $0 \leq x \leq 1$ and $p \in(-\infty,+\infty)$.

(2) The degenerate equation $F(p, x)=0$ has three roots $p=\varphi_{1}(x), p=\varphi_{2}(x)$, and $p=\varphi_{3}(x)$ such that $\varphi_{1}(x)<\varphi_{2}(x)<\varphi_{3}(x), 0 \leq x \leq 1$.

(3) The following inequalities

$$
\left.\frac{\partial F}{\partial p}\right|_{p=\varphi_{1}(x)}<0,\left.\quad \frac{\partial F}{\partial p}\right|_{p=\varphi_{2}(x)}>0,\left.\quad \frac{\partial F}{\partial p}\right|_{p=\varphi_{3}(x)}<0
$$

take place. 
(4) The equation

$$
\Phi(x)=\int_{\varphi_{1}(x)}^{\varphi_{3}(x)} F(p, x) d p=0
$$

has isolated root $x=x_{0}$ on the interval $0<x<1$.

Under these conditions,

(i) if $\Phi^{\prime}\left(x_{0}\right)<0$, then the solution $p(x, \varepsilon)$ of the problem (2) exists such that

$$
\lim _{\varepsilon \rightarrow 0} p(x, \varepsilon)= \begin{cases}\varphi_{3}(x), & 0<x<x_{0} \\ \varphi_{1}(x), & x_{0}<x<1\end{cases}
$$

and it is an asymptotically stable stationary solution of problem (1);

(ii) if $\Phi^{\prime}\left(x_{0}\right)>0$, solution $p(x, \varepsilon)$ of problem (2) exists such that

$$
\lim _{\varepsilon \rightarrow 0} p(x, \varepsilon)= \begin{cases}\varphi_{1}(x), & 0<x<x_{0} \\ \varphi_{3}(x), & x_{0}<x<1\end{cases}
$$

and it is an asymptotically stable stationary solution of problem (1).

The solutions that satisfy (5) or (6) are called the steplike contrasting structures or stationary fronts. There are also other stable stationary solutions of the problem (1). In particular, under Conditions 1-3 the existence of two more solutions, one of which is close to $\varphi_{1}(x)$ :

$$
\lim _{\varepsilon \rightarrow 0} p(x, \varepsilon)=\varphi_{1}(x), \quad 0<x<x_{0}
$$

and the other one is close to $\varphi_{3}(x)$ :

$$
\lim _{\varepsilon \rightarrow 0} p(x, \varepsilon)=\varphi_{3}(x), \quad 0<x<x_{0},
$$

is guaranteed.

There is an important problem of correspondence between a set of initial functions and a set of steady stationary solutions: given initial function $p^{0}(x)$, what steady-state solution will we have at $t \rightarrow+\infty$ ? And there is the inverse problem: if one of the steady states is more desirable than others, which conditions on $p^{0}(x)$ guarantee approach to this desirable steady state?

At last, when studying mathematical models of particular processes there is the following question which arises: if the existing $p^{0}(x)$ does not correspond to the desirably steady state, is it possible to change the right-hand part of (1) so that the solution would evolve to the desirable steady state?

This work is aimed at considering these problems for the "power-society" model which describes the dynamics of the power distribution in a hierarchy.

We base our study on the theory of contrasting structures [2-5], especially on the Butuzov-Nedelko theorem [13]. Some other issues related to nonstationary fronts were studied in [14-16].

\section{Nonstationary Fronts and Interpretation in the Nonlinear Singularly Perturbed "Power-Society" Model}

This section deals with mathematical modeling of the processes of power dynamics in the hierarchical structures. The model was firstly introduced by Mikhailov, 1994, and the books by Samarskii and Mikhailov 1997 and Mikhailov 2005 should also be mentioned.

Here the hierarchy is a ranked set of instances. Each instance has a particular set of powers. The amount of powers changes with time, and we call such variability the power dynamics. We suppose that there exists a numerical variable which specifies the amount of powers of a particular instance. The power dynamics appear through (a) the self-streamlining of the hierarchy and (b) the influence of the society.

Let us denote the rank of the instance in the hierarchy by $x$ so that $x=0$ at the top of the hierarchy and $x=1$ at the bottom. Denote by $p(x, t)$ the amount of powers of instance at time $t$.

The equation of the "power-society" model $[1,2]$ has the form (1), and $F(p, x)$ is called the reaction of a civil society. The paper [1] has shown that if $F(p, x)=-k_{1}\left(p-p_{0}(x)\right)$ (where $k_{1}=$ const $>0$ and the function $p_{0}(x)$ is the attractive power profile), then the solution $p=p_{0}(x)$ of the stationary degenerated equation $F(p, x)=0$ is stable. This means that the solution $p(x, t, \varepsilon)$ of (1) tends to $p_{0}(x)$ when $t \rightarrow+\infty$, $0<x<1$. So for sufficiently large values of $t$ the power profile is close to $p_{0}(x)$.

It was very important in the paper [1] that only one attractive profile is supposed to exist. Here we consider the case of two stable power profiles $\varphi_{1}(x)$ and $\varphi_{3}(x)$, and each of them is attractive. We call $\varphi_{1}(x)$ the participatory profile and $\varphi_{3}(x)$ the iron-hand profile. Both of them are stable due to inequalities (3).

Henceforth we consider the function $F(p, x)$ having the cubic nonlinearity. So we consider the equation

$$
\frac{\partial p}{\partial t}=\varepsilon^{2} \frac{\partial^{2} p}{\partial x^{2}}-k_{1}(x)\left(p-\varphi_{1}(x)\right)\left(p-\varphi_{2}(x)\right)\left(p-\varphi_{3}(x)\right)
$$

with boundary value conditions

$$
\left.\frac{\partial p}{\partial x}\right|_{x=0}=\left.\frac{\partial p}{\partial x}\right|_{x=1}=0
$$

The following conditions are supposed to hold.

Condition 1. The functions $k_{1}(x), \varphi_{i}(x), i=1,2,3$, have the continuous derivatives for $0 \leq x \leq 1$.

Condition 2. Inequalities $k_{1}(x)>0, \varphi_{1}(x)<\varphi_{2}(x)<\varphi_{3}(x)$ hold true for $0 \leq x \leq 1$.

We also notice that though $\varphi_{1}(x)>0$ because of the politological meaning of the function $\varphi_{i}(x)$, this condition must not be required from the mathematical point of view. 
Making the substitution (see [17])

$$
p=q \frac{\varphi_{3}(x)-\varphi_{1}(x)}{2}+\frac{\varphi_{3}(x)+\varphi_{1}(x)}{2},
$$

we obtain the equation for the function $u(x, t, \varepsilon)$ :

$$
\begin{aligned}
\frac{\partial q}{\partial t}= & \varepsilon^{2} \frac{\partial^{2} q}{\partial x^{2}}-\gamma(x)\left(q^{2}-1\right)(q-\varphi(x)) \\
& +\varepsilon^{2} \frac{\partial q}{\partial x} \Phi_{1}(x)+\varepsilon^{2} \Phi_{2}(q, x)
\end{aligned}
$$

Here

$$
\begin{gathered}
\gamma(x)=k_{1}(x)\left(\frac{\varphi_{3}(x)-\varphi_{1}(x)}{2}\right)^{2}>0, \\
\varphi(x)=\frac{2 \varphi_{2}(x)-\varphi_{3}(x)-\varphi_{1}(x)}{\varphi_{3}(x)-\varphi_{1}(x)}
\end{gathered}
$$

(notice that $-1<\varphi(x)<1$ ),

$$
\begin{gathered}
\Phi_{1}(x)=\frac{2\left(\varphi_{3}^{\prime}(x)-\varphi_{1}^{\prime}(x)\right)}{\varphi_{3}(x)-\varphi_{1}(x)}, \\
\Phi_{2}(q, x)=q \frac{\varphi_{3}^{\prime \prime}(x)-\varphi_{1}^{\prime \prime}(x)}{\varphi_{3}(x)-\varphi_{1}(x)}+\frac{\varphi_{3}^{\prime \prime}(x)+\varphi_{1}^{\prime \prime}(x)}{\varphi_{3}(x)-\varphi_{1}(x)} .
\end{gathered}
$$

Function $u(x, t, \varepsilon)$ satisfies boundary conditions

$$
\begin{aligned}
& {\left.\left[\varphi_{3}(0)-\varphi_{1}(0)\right] \frac{\partial q}{\partial x}\right|_{x=0}} \\
& +\left.\left[\varphi_{3}^{\prime}(0)-\varphi_{1}^{\prime}(0)\right] q\right|_{x=0}+\varphi_{3}^{\prime}(0)+\varphi_{1}^{\prime}(0)=0, \\
& {\left.\left[\varphi_{3}(1)-\varphi_{1}(1)\right] \frac{\partial q}{\partial x}\right|_{x=1}} \\
& +\left.\left[\varphi_{3}^{\prime}(1)-\varphi_{1}^{\prime}(1)\right] q\right|_{x=1}+\varphi_{3}^{\prime}(1)+\varphi_{1}^{\prime}(1)=0 .
\end{aligned}
$$

Consider stationary $(\partial / \partial t=0)$ equation related to $(12)$ :

$$
\begin{aligned}
\varepsilon^{2} q^{\prime \prime}= & \gamma(x)\left(q^{2}-1\right)(q-\varphi(x)) \\
& -\varepsilon^{2} \frac{\partial q}{\partial x} \Phi_{1}(x)-\varepsilon^{2} \Phi_{2}(q, x) .
\end{aligned}
$$

Using the boundary functions method [18] we construct the asymptotic contrast solution of the problem (16) and (15). The first-order asymptotic expansion has the form

$$
\begin{aligned}
q(x, t, \varepsilon)= & \bar{q}_{0}(x)+\varepsilon \bar{q}_{1}(x)+\Pi_{0} q(\tau) \\
& +\varepsilon \Pi_{1} q(\tau)+\varepsilon Q q\left(\tau_{0}, \tau_{1}\right),
\end{aligned}
$$

where $\bar{q}_{0}(x)$ and $\bar{q}_{1}(x)$ are the regular terms of asymptotic expansion, $\Pi_{0} q(\tau)$ and $\Pi_{1} q(\tau)$ are zero- and first-order transition layer functions, $\tau=\left(x-x_{*}\right) / \varepsilon$ is a stretched variable, $x_{*}=x_{*}(\varepsilon)$ is a transition point in a small vicinity of which the transition layer is localized, and $Q q\left(\tau_{0}, \tau_{1}\right)$ is function describing the boundary layers near the points $x=0, x=1$ and $\tau_{0}=x / \varepsilon, \tau_{1}=(1-x) / \varepsilon$. The transition point has the following asymptotic form:

$$
x_{*}=x_{0}+\varepsilon x_{1}+\varepsilon^{2} x_{2}+\cdots .
$$

Using the boundary functions method procedure $[3,4]$ we obtain that the principal term $x_{0}$ of the expansion (18) can be found from the equation

$$
\varphi\left(x_{0}\right)=0 .
$$

The full principal order function $\tilde{q}(\tau)=\bar{q}_{0}\left(x_{0}\right)+\Pi_{0} q(\tau)$ can be found from equation

$$
\begin{gathered}
\tilde{q}^{\prime \prime}=\gamma\left(x_{0}\right) \tilde{q}\left(\tilde{q}^{2}-1\right), \\
\tilde{q}(-\infty)=1, \quad \tilde{q}(+\infty)=-1 .
\end{gathered}
$$

From (20) and (21) we have

$$
\widetilde{q}=\frac{1-\exp \left[\sqrt{2 \gamma\left(x_{0}\right)} \tau\right]}{1+\exp \left[\sqrt{2 \gamma\left(x_{0}\right)} \tau\right]} .
$$

So the principal term of the stationary power profile has the form

$$
\begin{aligned}
p_{\mathrm{st}}(x, \varepsilon)= & \frac{1-\exp \left[\sqrt{2 \gamma\left(x_{0}\right)}\left(x-x_{0}\right) / \varepsilon\right]}{1+\exp \left[\sqrt{2 \gamma\left(x_{0}\right)}\left(x-x_{0}\right) / \varepsilon\right]} \\
& \times \frac{\varphi_{3}(x)-\varphi_{1}(x)}{2}+\frac{\varphi_{3}(x)+\varphi_{1}(x)}{2} .
\end{aligned}
$$

The power profile $p_{\mathrm{st}}(x, \varepsilon)$ is close to the iron-hand profile $\varphi_{3}(x)$ when $0 \leq x<x_{0}$ and to the participatory profile $\varphi_{1}(x)$ when $x_{0}<x \leq 1$. In the vicinity of the transition point $x_{0}$ we have $\partial p / \partial x \cong \varepsilon^{-1}$. We call such power profiles the contrast power profiles.

Equation (19) can be written in the form

$$
\varphi_{2}\left(x_{0}\right)=\frac{\varphi_{3}\left(x_{0}\right)+\varphi_{1}\left(x_{0}\right)}{2} .
$$

We call the function $h_{1}(x)=\varphi_{2}(x)-\varphi_{1}(x)$ the participatory domain's width and function $h_{3}(x)=\varphi_{3}(x)-\varphi_{2}(x)$ the iron-hand domain's width. Then (20) can be interpreted in the following way: at the transition point $x_{0}$ of the stationary contrast power profile (SCPP) the participatory domain's width is equal to the iron-hand domain's width $h_{1}\left(x_{0}\right)=h_{3}\left(x_{0}\right)$.

The stability of contrast structures of (9) was investigated by Bozhevol'nov and Nefëdov [5] and Vasil'eva et al. [6]. In terms of the "power-society" model the stability result can be interpreted as follows.

SCPP, which are close to the iron-hand profile at the top ranks of the hierarchy $\left(p \approx \varphi_{3}(x)\right.$ when $\left.0 \leq x<x_{0}\right)$ and to the participatory profile at the bottom ranks $\left(p \approx \varphi_{1}(x)\right.$ when $x_{0}<x \leq 1$ ), are stable if the iron-hand domain's width is greater than the participatory domain's width at the top ranks of the hierarchy $\left(h_{3}(x)>h_{1}(x)\right.$ when $\left.0 \leq x<x_{0}\right)$ and less at the bottom ranks $\left(h_{3}(x)<h_{1}(x)\right.$ when $\left.x_{0}<x \leq 1\right)$. If $h_{3}(x)<h_{1}(x)$ when $0 \leq x<x_{0}$ and $h_{3}(x)>h_{1}(x)$ when $x_{0}<x \leq 1$ then the SCPP is unstable. 
Remark 1. Similar statement holds for the so-called confederative SCPP which are close to the participatory profile at the top ranks of the hierarchy and to the iron-hand profile at the bottom ranks $\left(p \approx \varphi_{1}(x)\right.$ when $0 \leq x<x_{0}$ and $p \approx \varphi_{3}(x)$ when $\left.x_{0}<x \leq 1\right)$. They are stable if $h_{3}(x)<h_{1}(x)$ when $0 \leq x<x_{0}$ and $h_{3}(x)>h_{1}(x)$ when $x_{0}<x \leq 1$ and unstable if $h_{3}(x)>h_{1}(x)$ when $0 \leq x<x_{0}$ and $h_{3}(x)<h_{1}(x)$ when $x_{0}<x \leq 1$.

Consider again nonstationary equation (9). Suppose that at time $t=t_{0}$ contrasting structure has appeared with the transition layer at the vicinity of the point $x=\xi$. Then for $t>t_{0}$ the solution is a nonstationary contrast structure: $p \approx$ $\varphi_{3}(x)$ when $x<R(t, \varepsilon)$ and $p \approx \varphi_{1}(x)$ when $x>R(t, \varepsilon)$, where the transition point $R(t, \varepsilon)$ depends on time. We call such power profile the nonstationary contrast power profile (NCPP).

Let us construct the asymptotic NCPP.

Like in Section 3, make the substitution (6) and consider (7). It was shown in [7] that the principal term of the nonstationary contrast structure looks similar to one of the stationary contrast structure (18):

$$
\widehat{q}=\frac{1-\exp [\sqrt{2 \gamma(R)}(x-R) / \varepsilon]}{1+\exp [\sqrt{2 \gamma(R)}(x-R) / \varepsilon]},
$$

where the function $R=R(t, \varepsilon)$ can be found from the equation

$$
\frac{d R}{d t}=-\varepsilon \sqrt{2 \gamma(R)} \varphi(R) .
$$

So the principal term of NCPP has the form

$$
\begin{aligned}
p(x, t, \varepsilon)= & \frac{1-\exp [\sqrt{2 \gamma(R)}(x-R) / \varepsilon]}{1+\exp [\sqrt{2 \gamma(R)}(x-R) / \varepsilon]} \\
& \times \frac{\varphi_{3}(x)-\varphi_{1}(x)}{2}+\frac{\varphi_{3}(x)+\varphi_{1}(x)}{2} .
\end{aligned}
$$

Power profile $p(x, t, \varepsilon)$ is close to the iron-hand profile $\varphi_{3}(x)$ when $0 \leq x<R$ and to the participatory profile $\varphi_{1}(x)$ when $R<x \leq 1$. The value of $d R / d t$ represents the speed of the transition layer. In terms of the "power-society" model the expression for $d R / d t$ has the form

$$
\frac{d R}{d t}=-\varepsilon \sqrt{\frac{k_{1}(R)}{2}}\left[2 \varphi_{2}(R)-\varphi_{3}(R)-\varphi_{1}(R)\right]
$$

or

$$
\frac{d R}{d t}=\varepsilon \sqrt{\frac{k_{1}(R)}{2}}\left[h_{3}(R)-h_{1}(R)\right] .
$$

Consider now some important cases of using formula (29); see also [17].

2.1. Attraction to the "Iron-Hand" Profile (1). Let the "ironhand" domain's width be larger than participatory domain's width: $h_{3}(x)>h_{1}(x)$ for any $x \in[0,1]$. This means that the iron-hand profile looks more attractive from the society's point of view. Then SCPP do not exist because (24) has no roots. After appearing at time $t_{0}$ the contrast structure begins to move according to formula (29). Evidently $d R / d t>0$, and after small time of order $\varepsilon^{-1}$ transition point $R(t, \varepsilon)$ comes to the right end of the segment $[0,1]$. So the power profile appears close to the iron-hand profile for any $x \in[0,1]$. Notice that if at time $t=0$ function $p(x, 0, \varepsilon)$ is entirely in the participatory domain then for any $t$ the power profile is close to the participatory profile even if $h_{3}(x)>h_{1}(x)$. For appearing the power profile close to the iron-hand profile function $p(x, 0, \varepsilon)$ must be located in the iron-hand domain on at least one point in the interval $(0,1)$. This statement is based on the theorem proved by Bozhevol'nov and Nefedov $[5]$.

2.2. Attraction to the "Iron-Hand" Profile (2). Let point $x_{0} \in$ $(0,1)$ exist such that $h_{1}\left(x_{0}\right)=h_{3}\left(x_{0}\right), h_{1}(x)>h_{3}(x)$ when $x<x_{0}$ and $h_{1}(x)<h_{3}(x)$ when $x>x_{0}$. Then unstable SCPP exist having transition layer in the vicinity of the point $x_{0}$. Let function $p(x, 0, \varepsilon)$ be in the iron-hand domain for $x<\xi$ and in the participatory domain for $x>\xi$ where $x_{0}<\xi<1$. Then the power profile $p(x, t, \varepsilon)$ is attracted to the iron-hand profile for $x<\xi$ and to the participatory profile for $x>\xi$. After appearing at time $t_{0}$ the contrast structure begins to move according to formula (29) and initial condition $R(0, \varepsilon)=\xi$. As $\xi>x_{0}$ then $d R / d t>0$. So after small time of order $\varepsilon^{-1}$ transition point $R(t, \varepsilon)$ comes to the right end of the segment $[0,1]$. So the power profile appears close to the iron-hand profile for any $x \in[0,1]$.

2.3. Attraction to the Participatory Profile (1). Let $h_{3}(x)<$ $h_{1}(x)$ for any $x \in[0,1]$. This means that the participatory profile looks more attractive from the society's point of view. Then SCPP do not exist because (24) has no roots. After appearing at time $t_{0}$ the contrast structure begins to move according to formula (29). Evidently $d R / d t<0$ and after small time of order $\varepsilon^{-1}$ transition point $R(t, \varepsilon)$ comes to the left end of the segment $[0,1]$. So the power profile appears close to the participatory profile for any $x \in[0,1]$. Notice that if at time $t=0$ function $p(x, 0, \varepsilon)$ is entirely in the iron-hand domain then for any $t$ the power profile is close to the ironhand profile even if $h_{3}(x)>h_{1}(x)$. For appearing the power profile close to the participatory profile function $p(x, 0, \varepsilon)$ must be smooth and located in the participatory domain on at least one point in the interval $(0,1)$. This statement is based on the theorem proved by Bozhevol'nov and Nefedov $[5]$.

2.4. Attraction to the Participatory Profile (2). Let point $x_{0} \in$ $(0,1)$ exist such that $h_{1}\left(x_{0}\right)=h_{3}\left(x_{0}\right), h_{1}(x)>h_{3}(x)$ when $x<x_{0}$ and $h_{1}(x)<h_{3}(x)$ when $x>x_{0}$. Then unstable SCPP exist having transition layer in the vicinity of the point $x_{0}$. Let function $p(x, 0, \varepsilon)$ be in the iron-hand domain for $x<\xi$ and in the participatory domain for $x>\xi$ where $x_{0}<\xi<1$. Then according to (3) power profile $p(x, t, \varepsilon)$ is attracted to the iron-hand profile for $x<\xi$ and to the 
participatory profile for $x>\xi$. After appearing at time $t_{0}$ the contrast structure begins to move according to formula (26) and initial condition $R(0, \varepsilon)=\xi$. As $\xi<x_{0}$ then $d R / d t<0$. So after small time of order $\varepsilon^{-1}$ transition point $R(t, \varepsilon)$ comes to the left end of the segment $[0,1]$. So the power profile appears close to the participatory profile.

2.5. Attraction to SCPP. Let point $x_{0} \in(0,1)$ exist such that $h_{1}\left(x_{0}\right)=h_{3}\left(x_{0}\right), h_{1}(x)<h_{3}(x)$ when $x<x_{0}$ and $h_{1}(x)>$ $h_{3}(x)$ if $x>x_{0}$. Then the stable SCPP exist having transition layer in the vicinity of the point $x_{0}$. If there is $\xi \in(0,1)$ such that the initial function $p^{0}(x)$ satisfies $p^{0}(x)>\varphi_{2}(x)$, $0<x<\xi$, and $p^{0}(x)<\varphi_{2}(x), \xi<x<1$, then the contrasting structure appears after a short time $t_{0}$ such that $p(x) \approx \varphi_{3}(x)$ for $0<x<\xi$ and $p(x) \approx \varphi_{1}(x)$ for $\xi<x<1$. Then the contrasting structure begins to move according to formula (29) and initial condition $R\left(t_{0}, \varepsilon\right)=\xi$. So if $\xi<x_{0}$ then $d R / d t>0$ and if $\xi>x_{0}$ then $d R / d t<0$. So when $t \rightarrow \infty$, the transition point $R(t, \varepsilon)$ tends to the stationary transition point $x_{0}$. Thus the SCPP having transition layer in the vicinity of point $x_{0}$ appears.

\section{Parametric Optimization}

The total amount of power of the hierarchy is $\widetilde{P}(t, \varepsilon)=$ $\int_{0}^{1} p(x, t, \varepsilon)$. It was shown in [19] that there exists the optimal value $P_{0}$ of the total power which provides a maximum of steady-state consumption per capita (in frame of the "powersociety-economics" model [19]). So we should introduce the control parameter into the "power-society" model to make it controllable. So the problem would be to find the value of the control parameter under which $\widetilde{P}(t, \varepsilon) \rightarrow P_{0}$, when $t \rightarrow \infty$, $\varepsilon \rightarrow 0$.

Generally speaking, the model could be formulated such that the control is considered to be a function of time or $x$. In any case, the control describes the exogenous impact on the political system, such as a political pressure through media and political institutions. We restrict ourselves to the parametric control.

Definition 2 . The value $P_{0}$ is called the asymptotically achievable amount of the total power if there exists an admissible value of control parameter $u$ such that the steady-state total power $P_{u}(\varepsilon)$ satisfies $P_{u}(\varepsilon) \rightarrow P_{0}$ when $t \rightarrow \infty, \varepsilon \rightarrow 0$.

So consider the "power-society" model with nonlinear reaction of civil society:

$$
\begin{aligned}
f(p, x, t, u)= & -k_{1}(x)\left(p-\varphi_{1}(x)\right) \\
& \times\left(p-\left(\varphi_{2}(x)+\gamma u\right)\right)\left(p-\varphi_{3}(x)\right) .
\end{aligned}
$$

Here $k_{1}(x)>0$, the functions $k_{1}(x), \varphi_{1}(x), \varphi_{2}(x)$, and $\varphi_{3}(x)$ have continuous derivatives, and $\gamma$ is a constant. Thus the lowest and the biggest roots $\varphi_{1}(x), \varphi_{3}(x)$ of the degenerate equation $F(p, x, u)=0$ do not depend on the control, but there is an impact from the control to the "middle" $\operatorname{root} \varphi_{2}(x)$. So the model has the form

$$
\begin{aligned}
& \frac{\partial p}{\partial t}= \varepsilon^{2} \frac{\partial^{2} p}{\partial x^{2}}-k_{1}(x)\left(p-\varphi_{1}(x)\right) \\
& \times\left(p-\left(\varphi_{2}(x)+\gamma u\right)\right)\left(p-\varphi_{3}(x)\right), \\
&\left.\frac{\partial p}{\partial x}\right|_{x=0}=\left.\frac{\partial p}{\partial x}\right|_{x=1}=0,\left.\quad p\right|_{t=0}=p^{0}(x) .
\end{aligned}
$$

The initial function $p^{0}(x)$ is supposed to be smooth and satisfying $\varphi_{1}(x)<p^{0}(x)<\varphi_{3}(x)$. In other words, the initial distribution of power is between the iron-hand and participatory profiles.

The steady-state equation for (31) has the form

$$
\begin{gathered}
\varepsilon^{2} \frac{\partial^{2} p}{\partial x^{2}}=k_{1}(x)\left(p-\varphi_{1}(x)\right)\left(p-\left(\varphi_{2}(x)+\gamma u\right)\right)\left(p-\varphi_{3}(x)\right) \\
\left.\frac{\partial p}{\partial x}\right|_{x=0}=\left.\frac{\partial p}{\partial x}\right|_{x=1}=0
\end{gathered}
$$

Let us stress here that the control influences the relation between the width of the iron-hand domain and the width of the participatory domain.

Let the following conditions be fulfilled.

Condition 3. We have $-1 \leq u \leq 1$.

Condition 4. We have $\varphi_{1}(x)<\varphi_{2}(x)-\gamma<\varphi_{2}(x)+\gamma<\varphi_{3}(x)$ for $0 \leq x \leq 1$.

Condition 5. We have $H^{\prime}(x)<0$ for $0 \leq x \leq 1$, and here $H(x)=\varphi_{1}(x)+\varphi_{3}(x)-2 \varphi_{2}(x)$.

Conditions 3 and 4 introduce the normalization of the control such that for any admissible control $u \in[-1 ; 1]$ the $\operatorname{root} \varphi_{2}(x)+\gamma u$ is between the $\varphi_{1}(x)$ and $\varphi_{3}(x)$. The steadystate solution has no more than one transition point due to Condition 5.

If the control parameter $u$ is increased, the root of the equation $\varphi_{1}(x)+\varphi_{3}(x)-2\left(\varphi_{2}(x)+\gamma u\right)=0$ will move to the left. This means greater support to the participation ideas. If it exists. Analogically, the less the value of $u$ is, the more to the right the root of this equation is.

Consider the following problem. Let the desirable (optimal) value of total power be $P_{0}$. Is there a value of control parameter $u$, under which the steady-state solution (33) is such that $P_{u}(\varepsilon)=\int_{0}^{1} p(x, \varepsilon) d x \rightarrow P_{0}$ when $\varepsilon \rightarrow 0$ ?

From the practical point of view, such a formulation of the problem can be justified in the following way. We know from the "power-society-economics" model that the optimal value of the total power is some $P_{0}$, so we should try to tune the political system to provide this optimal value of power for the steady-state regime. 
Several cases should be distinguished.

Let us start our consideration from the situation in which both equations

$$
\begin{gathered}
\varphi_{1}(x)+\varphi_{3}(x)-2\left(\varphi_{2}(x)+\gamma\right)=0 \\
\varphi_{1}(x)+\varphi_{3}(x)-2\left(\varphi_{2}(x)-\gamma\right)=0
\end{gathered}
$$

have roots in the interval $(0 ; 1)$. Let us denote these roots by $a$ and $b$, respectively. Here we have $a<b$ in view of Condition 5.

The points $x=a$ and $x=b$ are the main asymptotic terms for the boundaries of the range within which the transition point of the stationary front is located.

Therefore, the value

$$
P_{a s}(u)=\lim _{\varepsilon \rightarrow 0} P(u, \varepsilon)=\lim _{\varepsilon \rightarrow 0} \int_{0}^{1} p(x, u, \varepsilon) d x
$$

satisfies the inequality

$$
\begin{aligned}
\int_{0}^{a} \varphi_{3}(x) d x+\int_{a}^{1} \varphi_{1}(x) d x & \leq P_{a s}(u) \\
& \leq \int_{0}^{b} \varphi_{3}(x) d x+\int_{b}^{1} \varphi_{1}(x) d x .
\end{aligned}
$$

Thus, in this case, $P_{0}$ is asymptotically achievable, if inequality

$$
\begin{aligned}
\int_{0}^{a} \varphi_{3}(x) d x+\int_{a}^{1} \varphi_{1}(x) d x & \leq P_{0} \\
& \leq \int_{0}^{b} \varphi_{3}(x) d x+\int_{b}^{1} \varphi_{1}(x) d x
\end{aligned}
$$

holds true.

The steady-state problem (32) and (33) has also solutions without transition layers: the iron-hand profile and the participatory one. So the values of total power

$$
P_{0}=\int_{0}^{1} \varphi_{3}(x) d x, \quad P_{0}=\int_{0}^{1} \varphi_{1}(x) d x,
$$

are also asymptotically achievable.

So, if both (34) and (35) have roots in the interval $(0 ; 1)$ then the set of asymptotically achievable values comprises the closed interval (38) and two isolated values (39): one of them is to the left of this closed interval, and the other one is to the right of it.

Now let us consider the situation in which (34) has a root $x=a \in(0 ; 1)$ and $(35)$ has no roots on $(0 ; 1)$.

In other words, $\varphi_{2}(x)-\gamma<\left(\varphi_{1}(x)+\varphi_{3}(x)\right) / 2$ for any $x$. So the values of $P_{0}$ in the closed interval

$$
\int_{0}^{a} \varphi_{3}(x) d x+\int_{a}^{1} \varphi_{1}(x) d x \leq P_{0} \leq \int_{0}^{1} \varphi_{3}(x) d x
$$

are asymptotically achievable.
Let now (35) have the root $x=b \in(0 ; 1)$, and let (34) have no roots in the interval. That is, at $u=1$, for any $x$, the middle root $\varphi_{2}(x)+\gamma$ is larger than half-sum of $\varphi_{1}(x)$ and $\varphi_{3}(x)$. Then the asymptotically achievable values are given by the inequality

$$
\int_{0}^{1} \varphi_{1}(x) d x \leq P_{0} \leq \int_{0}^{b} \varphi_{3}(x) d x+\int_{b}^{1} \varphi_{1}(x) d x .
$$

At last, consider the case in which neither of the equations has a root in the interval $(0 ; 1)$. Then the following three subcases are possible:

$$
\begin{aligned}
& \varphi_{1}(x)+\varphi_{3}(x)-2\left(\varphi_{2}(x)+\gamma\right)<0, \\
& \varphi_{1}(x)+\varphi_{3}(x)-2\left(\varphi_{2}(x)-\gamma\right)<0 \text { for any } x, \\
& \varphi_{1}(x)+\varphi_{3}(x)-2\left(\varphi_{2}(x)+\gamma\right)>0, \\
& \varphi_{1}(x)+\varphi_{3}(x)-2\left(\varphi_{2}(x)-\gamma\right)>0 \text { for any } x, \\
& \varphi_{1}(x)+\varphi_{3}(x)-2\left(\varphi_{2}(x)+\gamma\right)>0, \\
& \varphi_{1}(x)+\varphi_{3}(x)-2\left(\varphi_{2}(x)-\gamma\right)<0 \text { for any } x .
\end{aligned}
$$

It can be easily shown (see [2], e.g.) that in subcases (42) and (43) a steady-state front does not exist for any control parameter. So only the values of $P_{0}$ given by (39) are asymptotically achievable.

In the subcase (44) for any given $x_{0} \in(0 ; 1)$, such $u$ exists that the problem (32) and (33) has the stationary front with the transition point in the $\varepsilon$-vicinity of $x_{0}$. Therefore, any $P_{0}$ from closed interval

$$
\int_{0}^{1} \varphi_{1}(x) d x \leq P_{0} \leq \int_{0}^{1} \varphi_{3}(x) d x
$$

is asymptotically achievable.

The above speculations can be summarized as follows.

Theorem 3. Consider the problem (32) and (33) with parametric control. Let the following conditions be satisfied:

$$
\begin{aligned}
& \text { (1) } k_{1}(x), \varphi_{1}(x), \varphi_{2}(x), \varphi_{3}(x) \in C^{1}[0,1], \\
& \text { (2) }-1 \leq u \leq 1 \\
& \text { (3) } \varphi_{1}(x)<\varphi_{2}(x)-\gamma<\varphi_{2}(x)+\gamma<\varphi_{3}(x), \\
& \text { (4) } H^{\prime}(x)<0 \text {, where } H(x)=\varphi_{1}(x)+\varphi_{3}(x)-2 \varphi_{2}(x) .
\end{aligned}
$$

Then the set of asymptotically achievable values is not empty.

After the provided analysis of a steady-state problem (32) and (33), we go back to the initial parabolic partial problem (31) and (32).

Let some value $P_{0}$ be asymptotically achievable in the corresponding stationary problem. It means that there is a value of parametric control $u$, at which the problem (31), (32) has the steady-state solution for which the total power $P_{u}(\varepsilon)$ of the hierarchy asymptotically tends to $P_{0}$ when $\varepsilon \rightarrow 0$. However for the $\widetilde{P}_{u}(t, \varepsilon)=\int_{0}^{1} p_{u}(x, t, \varepsilon) d x$ we have $\widetilde{P}_{u}(t, \varepsilon) \rightarrow$ $P_{u}(\varepsilon)$ just for some class of initial functions $p^{0}(x)$. 
Thus, there is a problem to determine the class of initial power distributions for which, under the found value parametric control, the solution of the parabolic partial problem converges to the proper steady-state solution at $t \rightarrow \infty$.

The answer is given by the following theorem.

Theorem 4. (1) Let all the conditions of Theorem 3 be satisfied;

(2) let $P_{0}$ be an asymptotically achievable value of total power (denote by $u=u_{0}$ the corresponding value of parametric control);

(3) let the point $x_{0} \in(0 ; 1)$ exist such that

$$
P_{0}=\int_{0}^{x_{0}} \varphi_{3}(x) d x+\int_{x_{0}}^{1} \varphi_{1}(x) d x ;
$$

(4) let the points $x_{1} \in\left(0 ; x_{0}\right), x_{2} \in\left(x_{0} ; 1\right)$ exist such that

$$
p^{0}\left(x_{1}\right)>\varphi_{2}\left(x_{1}\right)+\gamma u_{0}, \quad p^{0}\left(x_{2}\right)<\varphi_{2}\left(x_{2}\right)+\gamma u_{0} .
$$

Then the solution $p_{u_{0}}(x, t, \varepsilon)$ of the parabolic partial problem (31) and (32) is such that the total power

$$
Q_{u_{0}}(t, \varepsilon)=\int_{0}^{1} p_{u_{0}}(x, t, \varepsilon) d x
$$

converges to

$$
P_{0}: \lim _{\varepsilon \rightarrow \infty} \lim _{t \rightarrow \infty} P\left(t, u_{0}, \varepsilon\right)=P_{0}
$$

Proof. It is easy to see that under these conditions the Butuzov-Nedelko theorem is fulfilled [13]. Therefore, at the chosen value of control $u=u_{0}$, the solution $p_{u_{0}}(x, t, \varepsilon)$ of the problem (31) and (32) has a passage to the limit

$$
\lim _{t \rightarrow \infty} p_{u_{0}}(x, t, \varepsilon)=p_{\text {st }, u_{0}}(x, \varepsilon),
$$

where $p_{\mathrm{st}}(x, \varepsilon)$ is the steady state solution for which

$$
\lim _{t \rightarrow \infty} p_{\text {st }, u_{0}}(x, \varepsilon)= \begin{cases}\varphi_{3}(x), & x<x_{0}, \\ \varphi_{1}(x), & x>x_{0} .\end{cases}
$$

By integrating (50) from $x=0$ to $x=1$ we get

$$
\lim _{t \rightarrow \infty} \int_{0}^{1} p_{u_{0}}(x, t, \varepsilon) d x=\int_{0}^{1} p_{\text {st }, u_{0}}(x, \varepsilon) d x .
$$

Passing to a limit $\varepsilon \rightarrow 0$, we get

$$
\begin{aligned}
\lim _{\varepsilon \rightarrow 0} \lim _{t \rightarrow \infty} \int_{0}^{1} p_{u_{0}}(x, t, \varepsilon) d x & =\lim _{\varepsilon \rightarrow 0} \int_{0}^{1} p_{\text {st }, u_{0}}(x, \varepsilon) d x \\
& =\int_{0}^{x_{0}} \varphi_{3}(x) d x+\int_{x_{0}}^{1} \varphi_{1}(x) d x \\
& =P_{0} .
\end{aligned}
$$

Thus $\lim _{\varepsilon \rightarrow 0} \lim _{t \rightarrow \infty} P\left(t, u_{0}, \varepsilon\right)=P_{0}$. Theorem 4 is proved.

\section{Conclusion}

It is shown that the theory of contrasting structures in singularly perturbed boundary value problems allows for investigating the properties of nonstationary fronts in the singularly perturbed "power-society" model. Depending on the initial condition, these fronts evolve to one of the asymptotically stable steady-state distributions of power within a government hierarchy.

There are some reasons to introduce a concept of desirable steady-state total amount of power of the hierarchy. The possibility theorem is proved for the problem of getting this amount by means of parametric control. The results can be used in investigating governing hierarchical systems.

\section{Conflict of Interests}

The authors declare that there is no conflict of interests regarding the publication of this paper.

\section{Acknowledgment}

This paper was partially supported by Russian Foundation for Basic Research (project 12-06-00205).

\section{References}

[1] V. F. Butuzov and A. B. Vasil'eva, "Asymptotic behavior of a solution of contrasting structure type," Akademiya Nauk SSSR, vol. 42, no. 6, pp. 831-841, 1987.

[2] V. F. Butuzov, A. B. Vasileva, and N. N. Nefedov, "Asymptotic theory of contrasting structures. A survey," Automation and Remote Control, vol. 58, no. 7, pp. 1068-1091, 1997.

[3] A. B. Vasil'eva, V. F. Butuzov, and N. N. Nefedov, "Singularly perturbed problems with boundary and internal layers," Proceedings of the Steklov Institute of Mathematics, vol. 268, no. 1, pp. 258-273, 2010.

[4] A. B. Vasil'eva, V. F. Butuzov, and N. N. Nefëdov, "Contrast structures in singularly perturbed problems," Fundamental'naya $i$ Prikladnaya Matematika, vol. 4, no. 3, pp. 799-851, 1998.

[5] Yu. V. Bozhevol'nov and N. N. Nefëdov, "Front motion in a parabolic reaction-diffusion problem," Computational Mathematics and Mathematical Physics, vol. 50, no. 2, pp. 264-273, 2010.

[6] A. Vasil'eva, A. Nikitin, and A. Petrov, "Stability of contrasting solutions of nonlinear hydromagnetic dynamo equations and magnetic fields reversals in galaxies," Geophysical and Astrophysical Fluid Dynamics, vol. 78, no. 1-4, pp. 261-279, 1994.

[7] D. Moss, A. Petrov, and D. Sokoloff, "The motion of magnetic fronts in spiral galaxies," Geophysical and Astrophysical Fluid Dynamics, vol. 92, no. 1-2, pp. 129-149, 2000.

[8] A. B. Vasil'eva, A. P. Petrov, and À. À. Plotnikov, "Alternating contrast structures," Computational Mathematics and Mathematical Physics, vol. 38, no. 9, pp. 1471-1480, 1998.

[9] A. P. Mikhailov, "Mathematical modeling of the authority in the hierarchical structures," Mathematical Modeling, vol. 6, article 6, 1994 (Russian).

[10] A. A. Samarskii and A. P. Mikhailov, Principles of mathematical modeling, Ideas, methods, examples, vol. 3 of Numerical Insights, Taylor \& Francis, London, UK, 2002. 
[11] A. P. Mikhailov, "Mathematical modeling of power distribution in state ierarchical structures interacting with civil society," in Proceedings of 14th IMACS World Congress, vol. 2, p. 828, Atlanta, Ga, USA, 1994.

[12] A. P. Mikhailov, "The research of the "Power-Society" system," Fizmatlit, 2006, (in Russian).

[13] V. F. Butuzov and I. V. Nedelko, "On the global influence domain of stable solutions with internal layers," Differential Equations, vol. 192, no. 5, pp. 13-52, 2001.

[14] N. N. Nefëdov and A. G. Nikitin, "The method of differential inequalities for step-type contrast structures in singularly perturbed integro-differential equations in the spatially twodimensional case," Differential Equations, vol. 42, no. 5, pp. 739749, 2006.

[15] N. N. Nefedov, A. G. Nikitin, M. A. Petrova, and L. Rekke, "Moving fronts in integro-parabolic reaction-advection-diffusion equations," Differential Equations, vol. 47, no. 9, pp. 1318-1332, 2011.

[16] N. N. Nefëdov and A. G. Nikitin, "Boundary and internal layers in the reaction-diffusion problem with a nonlocal inhibitor," Computational Mathematics and Mathematical Physics, vol. 51, no. 6, pp. 1011-1019, 2011.

[17] M. Dmitriev, A. Petrov, and G. Zhukova, "The nonlinear "Authority-Society" model," WSEAS Transactions on Mathematics, vol. 2, no. 1-2, pp. 31-36, 2003.

[18] A. B. Vasileva and V. F. Butuzov, "The method of boundary layer functions," Differential Equations, vol. 21, no. 10, pp. 1107-1112, 1985.

[19] M. G. Dmitriev, A. A. Pavlov, and A. P. Petrov, "Optimal power volume in social-economical hierarchy by consumption per capita criterion," Informacionnye Technologii i Vychislitelnye Sistemy, vol. 4, pp. 4-11, 2007 (Russian). 


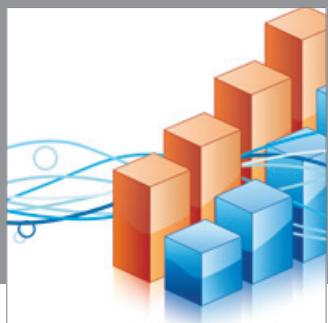

Advances in

Operations Research

mansans

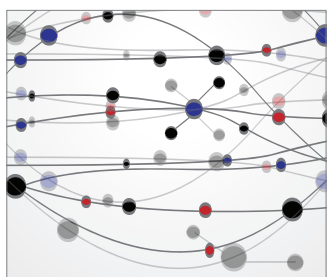

The Scientific World Journal
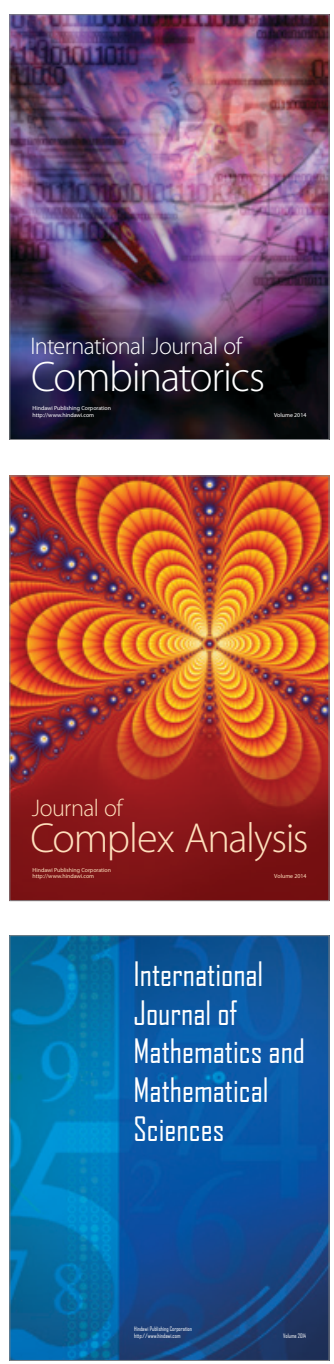
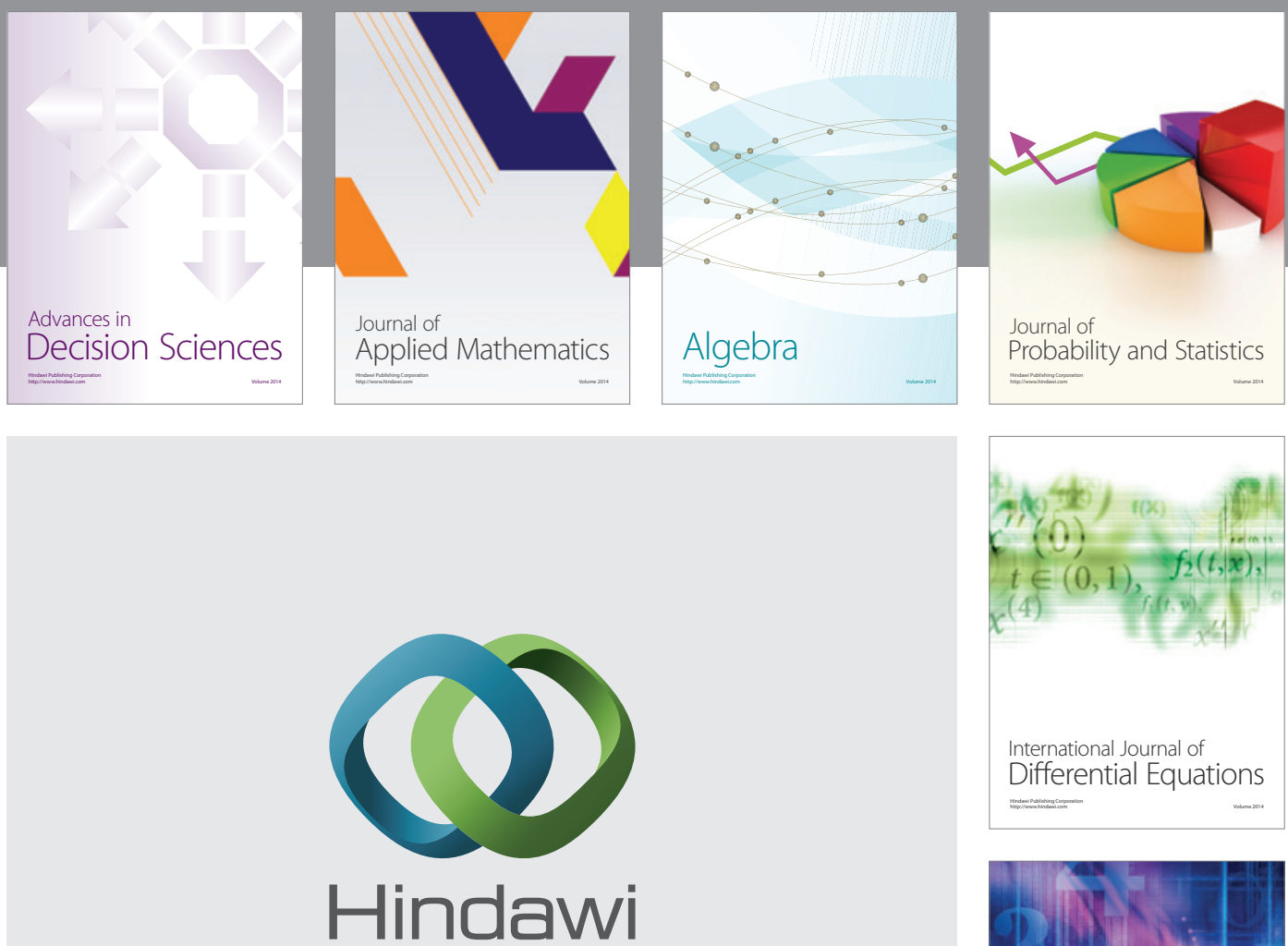

Submit your manuscripts at http://www.hindawi.com
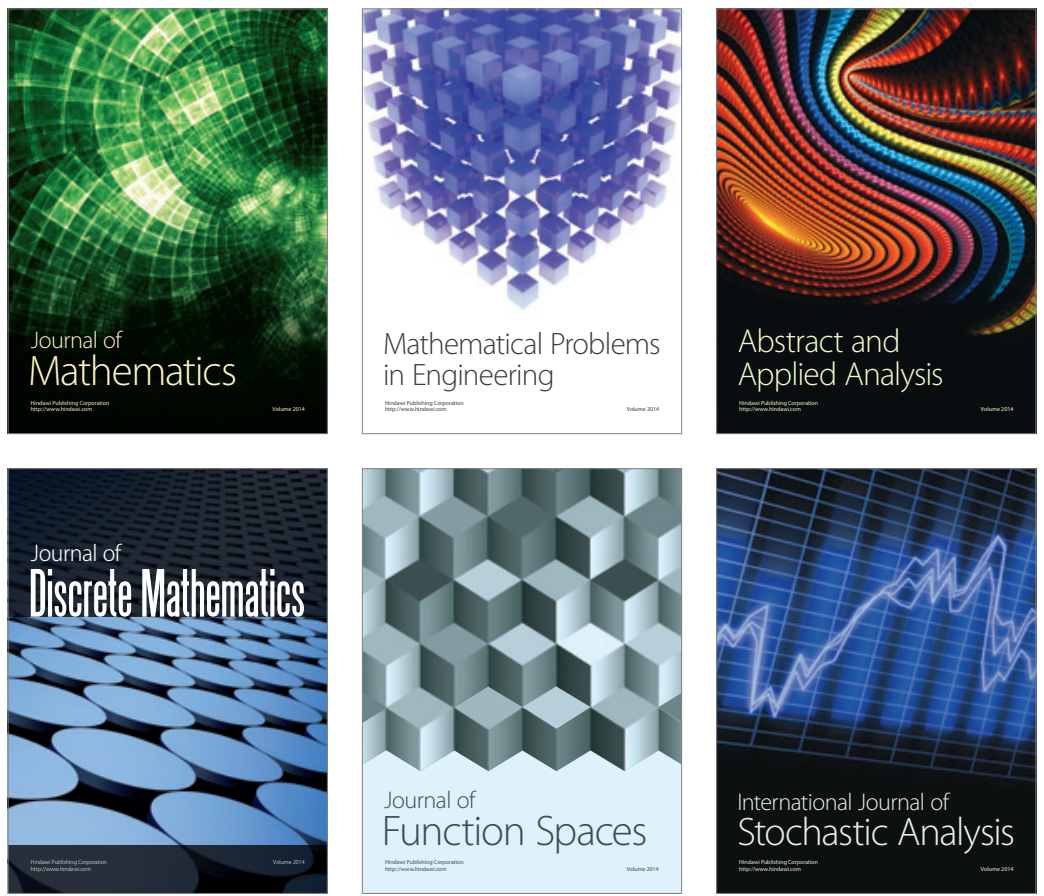

Journal of

Function Spaces

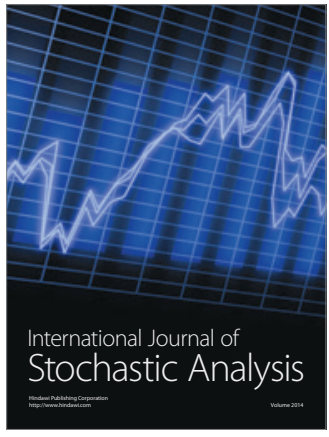

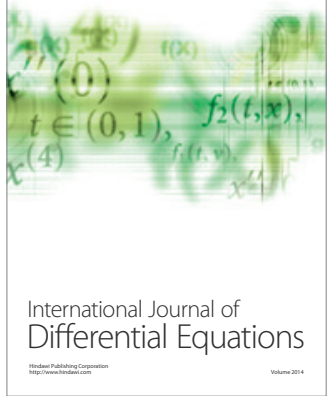
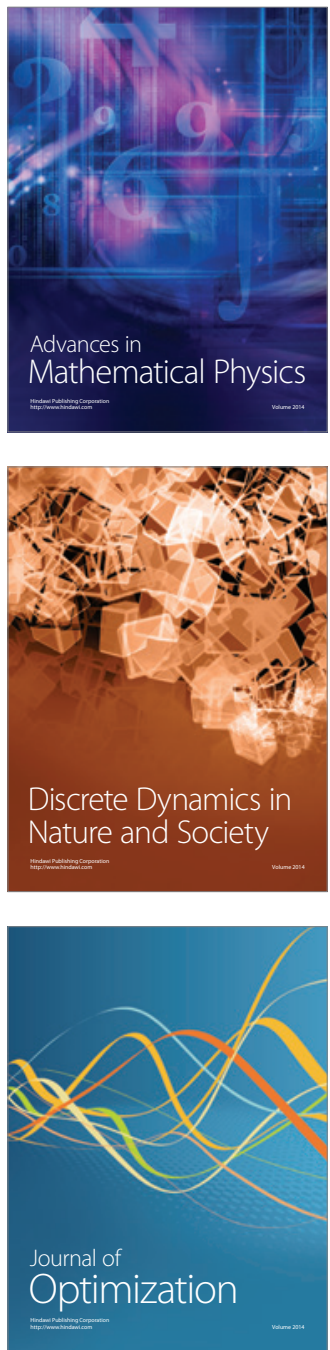